\title{
Integración de criterios de desempeños en el mejoramiento energético-ambiental de viviendas sociales existentes en Chile
}

\author{
Integration of performance criteria in the energy- \\ environmental improvement of exisiting social housing in \\ Chile
}

\section{Paulina Wegertseder Martínez Maureen Trebilcock Kelly}

\section{Resumen}

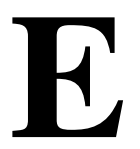

n Chile, $75 \%$ del parque habitacional existente fue construido sin consideraciones energéticas-ambientales, convirtiéndose en altos consumidores de energía. Hoy existen medidas de mejoramiento energético para viviendas existentes, pero éstas solo consideran algunos desempeños con implicancia en la demanda energética, siendo cada uno mejorado de manera independiente, sin considerar la relación y el efecto que conllevan al estar integrados. Esta investigación propone cubrir una brecha de conocimiento, de cómo diferentes criterios de desempeños actúan integralmente en el mejoramiento energético-ambiental de una vivienda. Actualmente se carece de tal conocimiento, que bajo la filosofía prestacional, genera diversas estrategias de mejoramiento para objetivos de desempeños en climas templados: Confort Térmico, Eficiencia Energética por Calefacción y Calidad del Aire Interior. Se somete un caso de estudio en el centro-sur de Chile, a un diagnostico post-ocupacional (monitorizaciones, simulaciones dinámicas y encuestas de percepción), para establecer criterios de desempeños, cuya relevancia e interdependencia se analiza mediante un estudio multifactorial. Se concluye que para alcanzar dichos objetivos es implícito mejorar la vivienda bajo la integración de criterios como: aumento de hermeticidad al aire, disminución del valor de transmitancia térmico y entrega del caudal de ventilación mínimo; siempre en una solución holística y no aislada.

Palavras-chaves: Mejoramiento energético-ambiental. Criterios de desempeños. Eficiencia energética. Confort térmico. Calidad del aire interior.

\begin{abstract}
In Chile, $75 \%$ of the existing housing stock was built without energyenvironmental considerations. These houses are becoming high energy consumers in the country. Nowadays, it exist different measures to improve the energy efficiency of existing dwellings, but these benchmarks consider only some performance that have implication on the energy demand. Normally, these performance are improving in an independently way, without considering the relationship between them and the effect that they imply when they are integrated. This research proposes to fill a gap in knowledge of how different performancebased behave integrally in the energy and environmental retrofitting of existing housing. Currently it is a lack of such knowledge, that under the performance based philosophy, generates various strategies for improve the final objective of performance-based in temperate climates: Thermal Comfort, Energy Efficiency for

Heating and Indoor Air Quality. It is selected a case study located in the centersouth of Chile. It is evaluated with a post-occupational diagnosis (monitoring, dynamics simulations and user perception surveys) to establish the performance criteria. Their relevance and interdependence are analyzed by a multifactorial study. We conclude that to achieve these goals is implicit to retrofit the existing housing under the integration of the following criteria: increased air tightness, decreased thermal transmittance value and delivery of the minimum ventilation rate; always in a comprehensive manner.
\end{abstract}

Paulina Wegertseder Martínez Universidad del Bío-Bío Concepcion - Chile

Maureen Trebilcock Kelly Universidad del Bío-Bío Concepcion - Chile

Recebido em 08/05/14 Aceito em 25/04/15
Keywords: Energy-environmental retrofit. Performance-based. Energy efficiency. Thermal comfort. Indoor air quality.

MARTÍNEZ, P. W.; KELLY, M. T. Integración de criterios de desempeños en el mejoramiento energético-ambiental de 47 viviendas sociales existentes en Chile. Ambiente Construído, Porto Alegre, v. 15, n. 2, p. 47-63, abr./jun. 2015. ISSN 1678-8621 Associação Nacional de Tecnologia do Ambiente Construído. http://dx.doi.org/10.1590/s1678-86212015000200013 


\section{Introducción}

A nivel mundial, calefaccionar una vivienda implica entre 40 y $60 \%$ del consumo energético medio, siendo el sector residencial el responsable de altas demandas de energía y del $40 \%$ de las emisiones de $\mathrm{CO}_{2}$ que recibe el planeta (INTERNATIONAL..., 2010). De acuerdo al Balance Nacional de Energía, el sector residencial consume $23 \%$ de la energía total en Chile (COMISIÓN..., 2011). Es posible disminuir estas alzas considerando una serie de medidas asociadas a una reducción de demanda de energía, así como la eficiencia energética. Se advierte que provocando cambios en el consumo energético del sector residencial, se puede beneficiar al país. Por lo cual, en el año 2000, el Estado implementa una medida para mejorar el confort habitacional de la población, creando el Artículo 4.1.10 (Reglamentación Térmica) de la O.G.U.C. (MINISTERIO..., 2006). Éste busca reducir y optimizar el consumo de energía por conceptos de calefacción y disminuir la contaminación intradomiciliaria en la vivienda nueva. En el año 2010, 74\% de viviendas no se encuentran acogidas a aquella normativa (INSTITUTO..., 2010), debido a su construcción previa al año 2000, resultando ser el causante de mayor consumo energético en el sector residencial, donde el usuario debe asumir el costo económico por el consumo energético, que supondría mantener la vivienda en condiciones de confort térmico.

Durante los últimos años, las fluctuaciones de precios en el mercado del gas y la electricidad, han provocado inestabilidades en el presupuesto familiar, afectando en mayor medida al sector más vulnerable. Personas que, en su mayoría, habitan viviendas sociales que no fueron construidas con objetivos de calidad energético-ambiental, sino que para satisfacer la necesidad de vivienda para personas que carecían de ella. En Chile, son miles las familias que viven bajo la línea de la "pobreza energética" (en inglés fuel poverty), causada por una combinación de bajos ingresos y altos costos de energía, además de una ineficiencia energética y mala calidad de la envolvente térmica de la vivienda (HONG et al., 2009).

Para dar solución a problemas energéticos y de mejoramiento de calidad en aquellas viviendas sin Reglamentación Térmica (RT), a partir del 2010, el Estado entrega US\$ 5.000 como subsidio de "Reacondicionamiento Térmico en Vivienda Existente", que mediante el cálculo del comportamiento térmico de la envolvente, propone soluciones de mejoramiento que cumplan con los estándares establecidos en la RT. Se exige que los complejos de techumbres, muros perimetrales y pisos inferiores ventilados, entendidos como elementos que constituyen la envolvente, deberán tener una transmitancia térmica (Valor U) igual o menor a la señalada para la zona que corresponda. Pero esta normativa no considera otros desempeños, que tienen influencia en el mejoramiento final, ya que no permite asegurar confort higrotérmico, alta hermeticidad ni otro tipo de desempeños. Si bien, la RT estableció un mayor estándar en parámetros que no eran exigidos, se hace necesario poder mejorar los valores propuestos e incluir nuevos desempeños que en la actualidad no son considerados al momento de diseñar y construir viviendas. Esta investigación tiene por objetivo cubrir una brecha de conocimiento sobre cómo diferentes criterios de desempeños actúan de forma integral en el mejoramiento energético-ambiental de viviendas existentes. Entendiendo "desempeño" como una integración de distintas variables o criterios, esta investigación se basa en la integración de diferentes desempeños asociados a cualidades de habitabilidad, durabilidad y sustentabilidad ambiental para lograr un mejor estándar en la vivienda, extendiendo su vida útil, y logrando un mayor confort para el usuario.

Actualmente ocurre que los mejoramientos se han desarrollado bajo guías de estrategias replicables, de carácter prescriptivo, coartando principalmente la innovación y la pertinencia de las estrategias al lugar. Por el contrario, si el carácter es prestacional en una rehabilitación, se deben establecer objetivos de desempeños y no pautas de mejoramiento, incentivando la creación y propuesta de estrategias que de manera prestacional alcanzan los estándares de desempeños. Esta falta de conocimiento impacta negativamente en la habitabilidad de las viviendas, con consecuencias sociales y económicas, debido a que las reformas en viviendas se generan de manera secuencial y no complementaria.

A partir del diagnóstico físico-constructivo y de confort térmico realizado en viviendas sociales existentes, contrapuesto con la percepción del usuario, se determinan que los desempeños que se deben estudiar y mejorar son tres: Confort Térmico, Eficiencia Energética por demanda de Calefacción y Calidad del Aire Interior. Pero no basta con conocer los desempeños finales, sino que se busca determinar cuáles son aquellas variables asociadas a ellos que son relevantes al momento de mejorar energética-ambientalmente una vivienda. Dichos parámetros son sometidos a un análisis multivariante, en función de la demanda energética 
para calefacción y del confort térmico. Asumiendo que se entregará el caudal de aire mínimo.

\section{Metodología}

\section{Descripción de los casos de estudio}

Se selecciona la comuna de Hualpén, donde $82 \%$ del parque habitacional construido corresponde a viviendas sociales. Ubicada en el centro-sur de Chile, en la Región del Bío-Bío, donde se evidencia una zona de transición bioclimática entre el clima mediterráneo del centro y el clima templado-frío, característico del sur. Haciendo uso de la clasificación empírica de las zonas climáticas de Köppen (INZUNZA, 2005), donde los climas son definidos por los valores medios anuales y mensuales de temperatura y de precipitación, se define el clima de Hualpén como: Templado Cálido con Estación Seca Corta (Csb) ${ }^{1}$.

Se realiza un catastro en la comuna, con información obtenida desde la Municipalidad. A partir de un método multi-factorial, se seleccionan, los cuatro casos más representativos (Figura 1). En relación a la forma de agrupación de las viviendas, existe una amplia tendencia hacia la agrupación pareada, siendo las aisladas las menos construidas, y las continuas de segunda preferencia. Del total construido, $80 \%$ presenta entre 30 y $50 \mathrm{~m}^{2}$ de superficie, donde $72 \%$ corresponde a construcciones de un nivel. La materialidad más usada es la albañilería y la madera.

En la Figura 2, se observan tres problemas recurrentes de viviendas sociales construidas en este clima. Dos corresponden a problemas físicoconstructivos: humedad y baja hermeticidad al aire de la envolvente, criterios que la actual normativa chilena no considera; y un tercero al hábito de secar la ropa en el interior, lo cual incrementa la humedad, provocando problemas en la calidad del aire interior y en la salud del habitante.

\section{Diagnóstico físico-constructivo}

El principal análisis para diagnosticar una vivienda existente es en base a la evaluación postocupacional, la cual se desarrolla bajo distintas técnicas. En este caso se abordan las técnicas comúnmente usadas en estudios de éste tipo, pero la diferencia está en que son aplicadas de forma paralela, permitiendo hacer una comparación de los resultados entregados.

${ }^{1} \mathrm{C}$ : Climas templados lluviosos. El mes más frío tiene una temperatura media comprendida entre $18^{\circ} \mathrm{C}$ y $-3^{\circ} \mathrm{C}$, y la media del mes más cálido supera los $10^{\circ} \mathrm{C}$; S: clima con estación seca en verano; y $\mathrm{B}$ : la temperatura media del mes más cálido es inferior a $22^{\circ} \mathrm{C}$, pero con temperaturas medias de al menos cuatro meses superiores a $10^{\circ}$.
El presente diagnóstico físico-constructivo incluye mediciones experimentales y simulaciones dinámicas (WEGERTSEDER, 2013). En complemento a esto, se realizaron encuestas para conocer la percepción de los usuarios.

Las mediciones fueron hechas para determinar ciertas propiedades físico-constructivas de la envolvente, de manera empírica. Se efectuó una medición de termo-flujometría de un muro exterior de la envolvente, que permite conocer el valor de transmitancia térmica de éste. Se registró también el nivel de hermeticidad al aire de la envolvente, mediante una prueba de despresurización (Blower Door Test).

Para las simulaciones dinámicas, se utiliza el software Design Builder y se determinan condiciones de borde iguales para todas las viviendas, con el fin de poder comparar el estado físico-constructivo de ellas, y no involucrar diferencias que ocurren al tener distintos usuarios, o regímenes de ventilación, etc. Se analiza la demanda energética por términos de calefacción y el confort térmico en dos períodos del año, los cuales son obtenidos desde el modelo adaptativo propuesto por la ASHRAE (AMERICAN..., 2010). Se especifican como: frio (abril-septiembre) y templado (octubre-marzo); para el primero, el rango de confort es entre 18 y $23^{\circ} \mathrm{C}$, en cambio para el templado se eleva entre 20 y $25^{\circ} \mathrm{C}$.

La encuesta desarrollada para conocer la percepción de los usuarios respecto al confort interior de sus viviendas es aplicada en las mismas dos temporadas del año, y es respondida por el total de habitantes del barrio con la tipología seleccionada.

Una vez conocido el diagnóstico de las viviendas, se comprueban los tres desempeños finales influyentes en el mejoramiento energéticoambiental de una vivienda. Luego, las variables relevantes para mejorar cada uno de éstos, son seleccionadas en función del resultado buscado: disminuir demanda de calefacción (sin aumentar la demanda de refrigeración), mejorar el confort térmico y optimizar la calidad del aire interior.

Se proponen distintos valores cuantitativos $y$ cualitativos para las variables seleccionadas, y son sometidas de manera integral en un estudio multifactorial, siendo cada combinación de variables representada por un resultado de simulación dinámica. 
Figura 1 - Características generales de los 4 casos de estudio seleccionados

\begin{tabular}{|c|c|c|c|}
\hline Cabo Aroca & Peñuelas II & Presidente Aylwin & El Triangulo \\
\hline 1989 & 1990 & 1993 & 1991 \\
\hline $30.36 \mathrm{~m}^{2}$ & $44.42 \mathrm{~m}^{2}$ & $40.38 \mathrm{~m}^{2}$ & $36,00 \mathrm{~m}_{2}$ \\
\hline $\begin{array}{c}\text { Estructura liviana en } \\
\text { madera }\end{array}$ & $\begin{array}{c}\text { Estructura en } \\
\text { Albañilería }\end{array}$ & $\begin{array}{l}\text { Estructura en } \\
\text { Albañilería }\end{array}$ & $\begin{array}{c}\text { Estructura liviana en } \\
\text { madera }\end{array}$ \\
\hline
\end{tabular}

Figura 2 - Imágenes interiores de las viviendas

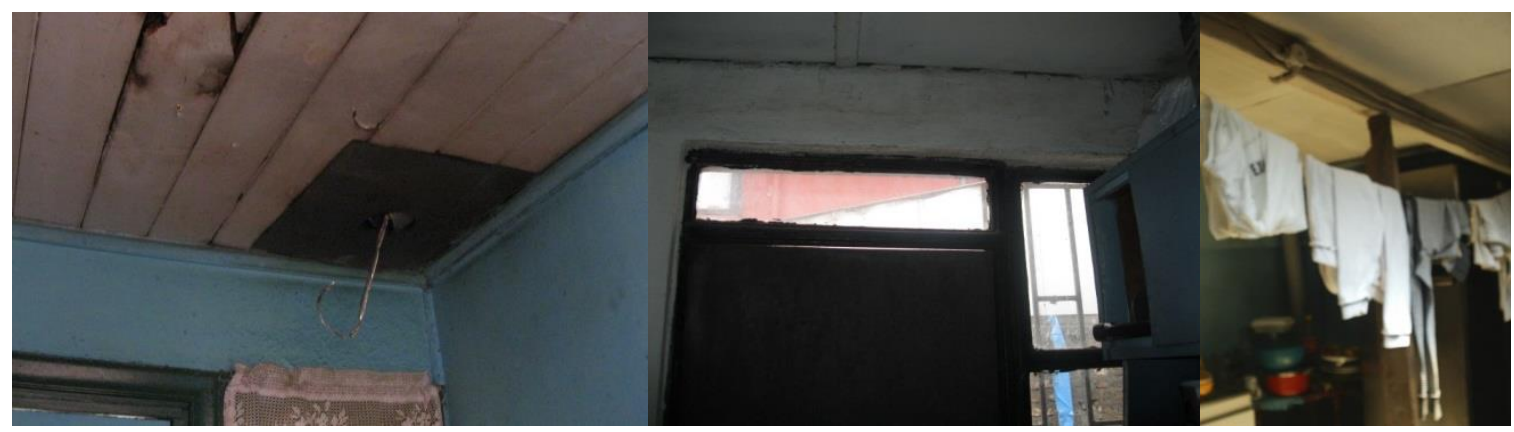

\section{Resultados del diagnóstico}

Los valores U monitoreados con termo-flujometría resultan entre 2,4 y $2,9 \mathrm{~W} / \mathrm{m}^{2} \mathrm{~K}$ para los casos de albañilería y 1,5 y $1,8 \mathrm{~W} / \mathrm{m}^{2} \mathrm{~K}$ en muros de madera. Estos últimos están más cerca de cumplir con lo exigido por la $\mathrm{RT}\left(1,7 \mathrm{~W} / \mathrm{m}^{2} \mathrm{~K}\right)$.

Los valores medidos de la tasa de infiltración de aire, distan ampliamente del valor establecido en otros casos o en estándares internacionales, donde la hermeticidad al aire de la envolvente resulta ser hasta cuatro veces inferior a lo obtenido en estas mediciones (Figura 3).

En las simulaciones dinámicas se involucraron los valores medidos experimentalmente, para así calibrar el modelo. Se obtiene que todas las viviendas presentan al menos $60 \%$ de las horas totales de la temporada cálida bajo la zona de confort térmico $\left(<20^{\circ} \mathrm{C}\right)$, donde los casos más desfavorables son los construidos en madera. Durante la temporada fría la situación es más perjudicial, ya que en todos los casos, $90 \%$ de las horas del año, la temperatura fluctúa bajo el rango de confort térmico, mostrando temperaturas de hasta $8^{\circ} \mathrm{C}$ en el interior.
La vivienda Cabo Aroca arroja una mayor demanda de energía, seguida por la vivienda Villa el Triángulo $\left(193,31 \mathrm{kWh} / \mathrm{m}^{2}\right.$ y $165,66 \mathrm{kWh} / \mathrm{m}^{2}$ respectivamente). Ambos casos de estudio están construidos con sistemas livianos de madera únicamente y son las viviendas que muestran mayores tasas de infiltraciones de aire. En cambio, las otras dos viviendas construidas en albañilería demandan en promedio $140 \mathrm{kWh} / \mathrm{m}^{2}$ al año.

En relación a las encuestas realizadas, el mayor desconfort térmico manifestado por los usuarios, ocurre en temporada fría, fenómeno que coincide con lo obtenido desde las simulaciones. En cambio para la temporada cálida, declaran estar en confort, aunque las simulaciones señalen lo contrario, arrojando gran porcentaje de temperaturas bajo la zona de confort. La percepción del usuario entrega indicios de que las mejoras deben ir orientadas hacia la temporada fría, donde el confort térmico es casi inexistente, sin poner en riesgo de sobrecaliento a la vivienda para la época estival.

A partir de la conjunción de todas las técnicas de diagnóstico realizadas, se seleccionaron ciertos desempeños que tuvieran influencia en los resultados de alta demanda energética por calefacción, de desconfort térmico durante los meses fríos y de mala calidad del aire. 
Figura 3 - Gráfico de Comparación de Estándares Internaciones y resultados obtenidos de los casos de estudio. Fuente. Elaboración propia

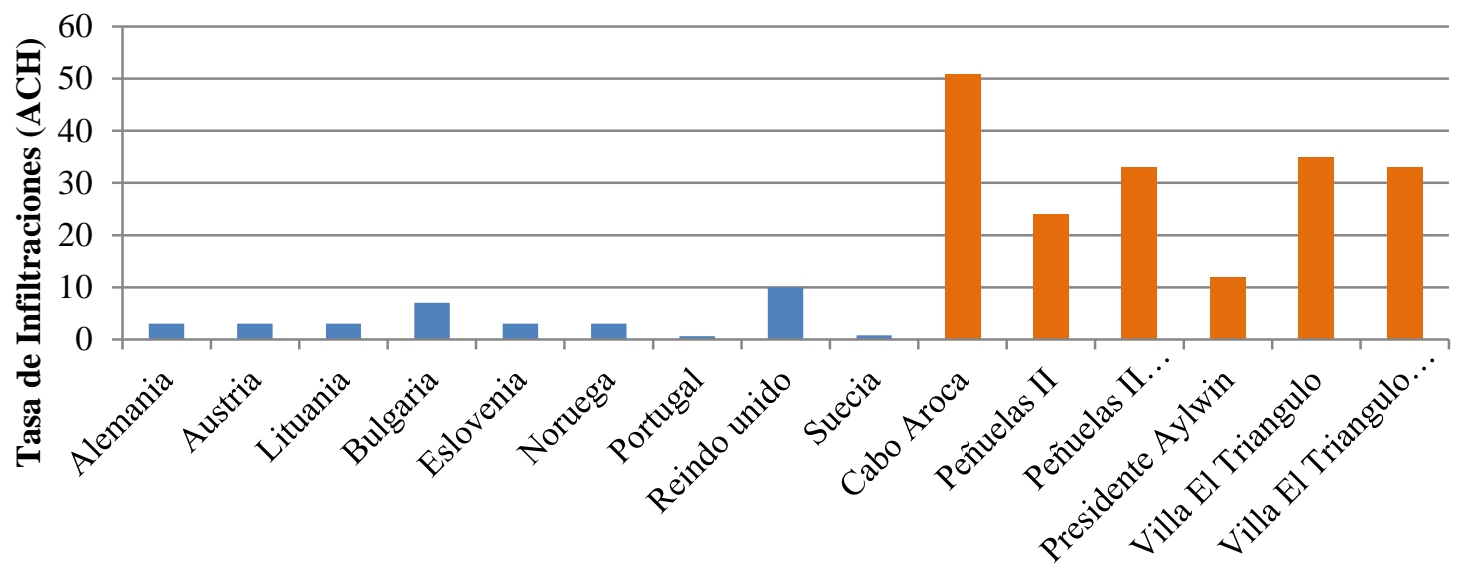

\section{Determinación de desempeños seleccionados}

Distintos sistemas de mejoramiento en viviendas existentes realizados en la última década (WILSON; SMITH; DUNN, 2007; MERCADO; ESTEVES; FILIPPÍN, 2010; CAUBERGHUYGEN..., 2009; HOWDEN-CHAPMAN et al., 2005), consideran desempeños como la eficiencia energética y la calidad térmica del edificio. Abordan parámetros como la contaminación al interior de la vivienda, la temperatura interior y la aislación térmica de la envolvente; pero no consideran otros, que la presente investigación si evalúa: aislación higrotérmica y hermeticidad al aire de la envolvente; los cuales además son desempeños que la actual RT no exige.

La metodología aplicada para definir los desempeños, y sus variables a considerar, establece cuatro pasos (NORDIC..., 1978):

(a) objetivos finales de desempeño;

(b) estándares de desempeños (indicadores y desempeños intermedios);

(c) métodos de verificación; y

(d) estrategias de referencia.

Este modelo se rige bajo un carácter prestacional, ya que establece el objetivo en los criterios de desempeños y no en alguna estrategia en particular.

Los criterios de desempeños representan objetivos finales dentro del mejoramiento energéticoambiental, y son identificados por ciertos estándares que se establecen como requisitos mínimos a cumplir. Para esta investigación se definen desempeños de orden cuantitativo y se propone trabajar con estrategias basadas en criterios prestacionales, y no prescriptivos, como comúnmente se hace en mejoramientos de edificaciones. Esta investigación no busca replicar estrategias prescriptivas aludiendo a una guía o cookbook (LEE; BARRETT, 2003), si no que a establecer, de manera prestacional, criterios de desempeños para mejorar energéticoambientalmente las viviendas sociales del clima en estudio.

Los desempeños a evaluar suelen ser fenómenos multidimensionales, que requieren un enfoque multidimensional, el cual contenga distintos y eficaces indicadores de rendimiento acorde al desempeño medido (MWASHA; WILLIAMS; IWARO, 2011). Estos fenómenos están determinados por variados parámetros, que tienen incidencia en el desempeño final, y son definidos en función de los resultados buscados, los cuales dentro de un estudio multifactorial son denominados como "variable respuesta", y en este caso corresponden a:

(a) disminuir demanda de calefacción; y

(b) Aumentar el confort térmico en el interior de la vivienda.

El tercer desempeño, calidad del aire interior, fue considerado como un criterio mínimo a cumplir, estableciéndolo por defecto en las simulaciones, un caudal mínimo de $1 \mathrm{l} / \mathrm{s} \mathrm{m}^{2}{ }^{2}$, asegurando una correcta ventilación.

Los desempeños "Confort térmico" y "Eficiencia Energética" fueron agrupados, ya que ambos se ven influenciados por los mismos criterios a

\footnotetext{
${ }^{2}$ Para determinar el caudal mínimo de aire, se utiliza de referencia la ASHRAE, que exige 7,5 l/s por persona, el cual es transformado a la unidad de medida requerida por DB (l/s m2). El valor más exigente resultado fue $0,89 \mathrm{l} / \mathrm{s} \mathrm{m}^{2}$, lo cual permite concluir que el valor a utilizar como estándar en todos los casos será de $1 \mathrm{l} / \mathrm{s} \mathrm{m}^{2}$, debido a su aproximación más eficiente.
} 
mejorar. En lo Cuadro 1, se muestran los indicadores de objetivos finales de desempeños, los desempeños intermedios que se deben considerar para alcanzarlos, sus respectivos indicadores, variables de diseño que se propusieron para medir el cumplimiento de los estándares finales, y los métodos de verificación. Estos últimos deben ser realizados antes del análisis del estado actual de la vivienda, para poder calibrar las simulaciones, y obtener resultados cercanos a la realidad, y así definir estrategias con mayor beneficio. Se recomienda realizar mediciones post-mejoramiento, para corroborar que los criterios de desempeño se hayan alcanzado.

Las variables de diseño que se intervienen corresponden solo a aquellas que puedan ser modificables, no es posible considerar otras variables, como la ubicación de la vivienda por ejemplo, que solo es modificable en construcciones nuevas.

Los valores (niveles) de los requisitos de desempeños, se determinan sin la necesidad de involucrar una estrategia constructiva en particular, aludiendo al carácter prestacional de la investigación. Los niveles fueron variados, algunos extraídos desde el diagnostico experimental, otros correspondientes a lo exigido en la RT y los demás corresponden a mejoramientos propuestos. Esta medida busca reflejar la influencia de la condición real del edificio.

Para el desempeño intermedio de aislación higrotérmica de la envolvente, se determina que el riesgo de condensación superficial va relacionado con el valor $U$ del muro y ventana. Para conocer qué valor disminuirá aquel riesgo, se realizan cálculos que determinan que al ser la transmitancia térmica del muro igual, o inferior, a $0,8 \mathrm{~W} / \mathrm{m}^{2} \mathrm{~K}$, no existirá riesgo de condensación superficial bajo las condiciones de borde establecidas en la Tabla 1, las cuales son especificas hacia realidades desfavorables de la vivienda social, donde la humedad interior es muy alta debido mala calidad de la construcción y hábitos del usuario.

Cuadro 1 - Desempeños finales e intermedios con sus respectivos indicadores, variables de diseño y métodos de verificación

\begin{tabular}{|c|c|c|c|c|c|}
\hline $\begin{array}{l}\text { Objetivo de } \\
\text { Desempeños }\end{array}$ & $\begin{array}{l}\text { Indicador de } \\
\text { Objetivos de } \\
\text { Desempeños }\end{array}$ & $\begin{array}{l}\text { Desempeños } \\
\text { Intermedios } \\
\text { (Requisitos } \\
\text { funcionales) }\end{array}$ & $\begin{array}{c}\text { Indicador } \\
\text { de } \\
\text { Desempeños } \\
\text { Intermedios }\end{array}$ & $\begin{array}{c}\text { Variable de } \\
\text { Diseño } \\
\text { (Requisitos de } \\
\text { Desempeños) }\end{array}$ & $\begin{array}{l}\text { Método de } \\
\text { verificación }\end{array}$ \\
\hline \multirow{3}{*}{$\begin{array}{l}\text { Confort } \\
\text { Térmico }\end{array}$} & \multirow{3}{*}{$\begin{array}{l}\text { Distribución } \\
\text { de Frecuencia } \\
\text { de } \\
\text { Temperatura } \\
(\%)\end{array}$} & $\begin{array}{l}\text { Transmitancia } \\
\text { Térmica de la } \\
\text { Envolvente }\end{array}$ & $\mathrm{W} / \mathrm{m}^{2} \mathrm{~K}$ & $\begin{array}{l}\text { Valor U Muro, } \\
\text { techo, piso y } \\
\text { vidrio }\end{array}$ & $\begin{array}{l}\text { Termoflujometría } \\
\text { según ASTM 518- } \\
10\end{array}$ \\
\hline & & \multirow[t]{2}{*}{$\begin{array}{l}\text { Aislación } \\
\text { higrotérmica } \\
\text { de la } \\
\text { envolvente }\end{array}$} & $\begin{array}{l}f \text { Rsi }(\mathrm{sd}) / \\
\text { fRsimin }< \\
\text { fRsi }\end{array}$ & $\begin{array}{l}\text { Ocurrencia de } \\
\text { condensación } \\
\text { superficial }\end{array}$ & $\begin{array}{l}\text { Monitorización de } \\
\text { variables de } \\
\text { temperatura y } \\
\text { humedad relativa } \\
\text { del aire según } \\
\text { protocolo de } \\
\text { medición }\end{array}$ \\
\hline & & & $\mathrm{p}(\mathrm{Pa})$ & $\begin{array}{l}\text { Ocurrencia de } \\
\text { condensación } \\
\text { intersticial }\end{array}$ & $\begin{array}{l}\text { Simulación de la } \\
\text { solución del muro } \\
\text { con software } \\
\text { aceptado }\end{array}$ \\
\hline \multirow[t]{2}{*}{$\begin{array}{l}\text { Eficiencia } \\
\text { Energética }\end{array}$} & $\begin{array}{l}\text { Energética } \\
\text { para } \\
\text { Calefacción } \\
\left(\mathrm{kWh} / \mathrm{m}^{2} \text { año }\right)\end{array}$ & $\begin{array}{l}\text { Hermeticidad } \\
\text { al aire de } \\
\text { envolvente }\end{array}$ & $\mathrm{ACH}$ & $\begin{array}{l}\text { Tasa de } \\
\text { Infiltraciones de } \\
\text { aire }\end{array}$ & $\begin{array}{l}\text { Presurización } \\
\text { según } \\
\text { UNE13728:2003 } \\
\text { (Blower Door). } \\
\end{array}$ \\
\hline & & $\begin{array}{l}\text { Cantidad de } \\
\text { Superficie } \\
\text { vidriada }\end{array}$ & $\mathrm{m}^{2}$ & $\begin{array}{l}\text { Porcentaje } \\
\text { superficie } \\
\text { vidriada hacia el } \\
\text { norte }\end{array}$ & Levantamiento \\
\hline $\begin{array}{l}\text { Calidad del } \\
\text { Aire Interior }\end{array}$ & $\begin{array}{l}\text { Tasa de Aire } \\
\text { Interior } 1 / \mathrm{s} \\
\text { por persona o } \\
1 / \mathrm{s} \text { por } \mathrm{m}^{2}\end{array}$ & - & - & $\begin{array}{l}\text { Caudal de } \\
\text { ventilación } \\
\text { mínimo exigido }\end{array}$ & $\begin{array}{l}\text { Monitorización de } \\
\text { concentración de } \\
\mathrm{CO}_{2}\end{array}$ \\
\hline
\end{tabular}

52 Martínez, P. W.; Kelly, M. T. 
Tabla 1 - Humedad relativa y Temperatura de aire interior y exterior, para criterio de desempeño

\begin{tabular}{cccc}
\hline $\begin{array}{c}\text { Temperatura de Aire } \\
\text { Exterior }(\boldsymbol{\theta e})\left({ }^{\circ} \mathrm{C}\right)\end{array}$ & $\begin{array}{c}\text { Temperatura de Aire } \\
\text { Interior }\left(\boldsymbol{\theta} \text { i) }\left({ }^{\circ} \mathbf{C}\right)\right.\end{array}$ & $\begin{array}{c}\text { Humedad Relativa } \\
\text { Exterior }(\varphi \mathrm{e})(\boldsymbol{\%})\end{array}$ & $\begin{array}{c}\text { Humedad Relativa } \\
\text { Interior }(\boldsymbol{\varphi} \mathbf{i})(\%)\end{array}$ \\
\hline 5,6 & 18,0 & 87,0 & 90 \\
\hline
\end{tabular}

Para asegurar el caudal de aire mínimo para ventilación se propone la utilización de un sistema de ventilación, el cual además cuenta con un sistema de recuperación de calor (HRV) de alto rendimiento $(70 \%)$, y con sistema de by-pass para temporada de verano, evitando un sobrecalentamiento cuando la temperatura aumenta. Esto es solo una posibilidad del sistema, que permite visualizar la influencia de la incorporación de él. Este sistema, además de asegurar un caudal mínimo de aire, permite ahorrar energía para calefacción, disminuyendo el uso de fuentes de calor, debido al calentamiento del aire recuperado. Además, ingresa el aire filtrado y limpio, evitando mayor contaminación al interior de la vivienda.

El uso de este tipo de sistemas de ventilación con HRV requiere de una alta hermeticidad en la envolvente, asegurando un mínimo intercambio de aire por infiltración, por lo tanto se hace más necesaria la renovación del aire por ventilación propuesta.

\section{Análisis multifactorial}

Para analizar la influencia real de cada parámetro, y así definir los criterios de desempeños finales, se propone como metodología un análisis multifactorial, el cual corresponde a una técnica de estudio multivariante, que sirve para interpretar las correlaciones entre un grupo de variables, donde existen factores que intervienen de menor, o mayor, manera en el resultado buscado (OLOFSSONANDERSSON; SJÖGREN, 2009). El efecto de cada factor se define como el cambio en la respuesta producido por un cambio en el valor del factor (MONTGOMERY, 2003).

Estudiar distintas y múltiples estrategias de mejoramiento en una misma edificación es crucial para reducir el consumo de energía. Existen ciertos parámetros que debieran tener mayor relevancia en la reducción del consumo energético, como por ejemplo la tasa de infiltración de aire, donde hasta una mínima diferencia entre 0 y $0,2 \mathrm{ACH}$ puede influir fuertemente en la demanda de energía (HOPFE; HENSEN, 2011). La actual Calificación Energética (CE) chilena, califica las viviendas considerando $1 \mathrm{ACH}$ como tasa, pero distintas mediciones de hermeticidad al aire de la envolvente (FIGUEROA et al., 2013) muestran valores que fluctúan entre $1,75 \mathrm{ACH}$ para una vivienda de albañilería, y hasta 2,49 $\mathrm{ACH}$ para una de madera, haciendo suponer que el uso de $1 \mathrm{ACH}$ constante como infiltración no es recomendable.

Del análisis multifactorial resulta una matriz, donde cada combinación de parámetros se ve representada por un punto, y responde cuál de ellas es la más óptima según el resultado buscado. Cada punto es una combinación lineal de las variables originales con coeficientes dados por el factor inicial, que finalmente, entrega una mejor compresión de la relación causa - efecto (LAM et al., 2010).

Como la cantidad de niveles y parámetros propuestas son variadas, aumenta el número de simulaciones, como así también la complejidad de cambiar manualmente cada parámetro en un software de simulación, haciendo que el tiempo necesario para simular crezca exponencialmente. Para el caso de estudio Cabo Aroca, por ejemplo, la cantidad de simulaciones crece a 6.336. Para evitar los cambios manuales de cada nivel, se optimiza el trabajo usando el sistema automatizador de simulaciones GenOpt, el cual permite realizar estudios paramétricos, entregando una base de datos completa con un resultado por cada cambio de nivel de factor. GenOpt evalúa la variable de respuesta con un programa de simulación externo, pudiéndose conectar a cualquier programa de simulación que lea los datos ingresados como archivos de texto. En este caso, se simula con el motor de Energy Plus, el mismo que se utilizó en la etapa de diagnóstico.

Para efectuar un análisis basado en parámetros, se realizó una lista, tipo ranking, donde se ordenaron los parámetros según su influencia en los resultados previstos (TAVARES; MARTINS, 2007; HEISELBERG et al., 2010; HEO; CHOUDHARY; AUGENBROE, 2012). Estos datos se analizan en función de objetivos los desempeños: demanda energética por calefacción y confort térmico por frecuencia de temperatura.

\section{Análisis en función de la demanda energética}

Para el análisis del desempeño de eficiencia energética por calefacción, se utiliza como referente la $\mathrm{CE}$ de Chile, siendo un parámetro de 
análisis significativo que atiende exclusivamente este desempeño. Permite conocer rangos de ahorro energético, donde se identifica la influencia que tienen las variables de diseño para los desempeños finales.

La CE utiliza 1 ACH como tasa de infiltración de aire constante, en cambio para los mejoramientos propuestos para los casos de estudios, se utilizan distintos valores que descienden desde la tasa medida insitu. Debido a esto, se simularon las viviendas con una renovación de aire por hora también, para comparar la diferencia causada al usar infiltraciones medidas o estimadas. A diferencia de la calificación, no se usa la herramienta de cálculo propuesta por la $\mathrm{CE}$, sino que las simulaciones energéticas con EnergyPlus.

Se recurre a los mismos rangos de demanda energética para calefacción entregados por la $\mathrm{CE}$ (A a G, siendo la A la más eficiente, y E el mínimo para calificar como eficiente), para evaluar las distintas combinaciones de variables que se propusieron para cada caso. Las demandas arrojadas por la vivienda mejorada se comparan con otra vivienda de las mismas características pero sin los mejoramientos, la cual solo debe cumplir con la RT y es calificada como E. Esta vivienda E resulta ser el caso base y a partir de ella las viviendas con mejoramientos ya pueden ser calificadas como más eficientes.

\section{Resultados calificados según rangos A a $\mathrm{G}$}

De los resultados obtenidos post-mejoramiento, se obtiene que los cuatro casos de estudio alcanzan una calificación A, reduciendo hasta $80 \%$ la demanda energética por términos de calefacción, en relación a la inicial (Figura 4).

El método de análisis consta en dos calificaciones de demanda energética por calefacción para cada vivienda, una utilizando diferentes tasas de infiltración de aire y otra sólo con $1 \mathrm{ACH}$. Debido a la extensión de los resultados de los cuatro casos de estudio, se selecciona un solo caso para mostrar en detalle: Peñuelas II.

Se exponen tres gráficos, el primero (Figura 5) corresponde a los resultados de demanda energética obtenidos de la vivienda existente con las estrategias de mejoramiento propuestas en base a los parámetros seleccionados. En él se considera la tasa de infiltración real y sus correspondientes mejoramientos. Para realizar una comparación general, se destacan en él los valores de demanda energética por calefacción para el caso real existente, el caso base con tasa de infiltración 1 ACH (E1) y el caso base con la tasa medida (E2). Del primer gráfico se desprende una tabla, donde se indica la tendencia de parámetros más usados en cada estrategia.

En un segundo gráfico (Figura 6, izq.) se exponen las demandas energéticas por calefacción, considerando todos los niveles de mejoramiento, a excepción de la hermeticidad al aire, la cual es definida como $1 \mathrm{ACH}$, tal como establece la $\mathrm{CE}$. El tercer gráfico (Figura 6, der.) si considera la infiltración al aire, logrando comparar la demanda energética al considerar la hermeticidad al aire medida.

La estrategia que cumple con la RT, considerando $1 \mathrm{ACH}$, demanda $106,19 \mathrm{kWh} / \mathrm{m}^{2}$ año de energía en calefacción (E1), es decir disminuye 16\% la demanda actual. El caso base E2 disminuye 11\% la demanda. La diferencia entre ambas es menor, por lo que al mantener todos las otras variables iguales para ambos casos, no valdría el esfuerzo disminuir la tasa de infiltración de aire a $1 \mathrm{ACH}$ (Figura 5). En esto influye también que la tasa de infiltración de aire inicial de esta vivienda no era tan alta $(1,23$ $\mathrm{ACH})$.

Figura 4 - Comparación de demanda energética por calefacción: caso inicial v/s caso mejorado

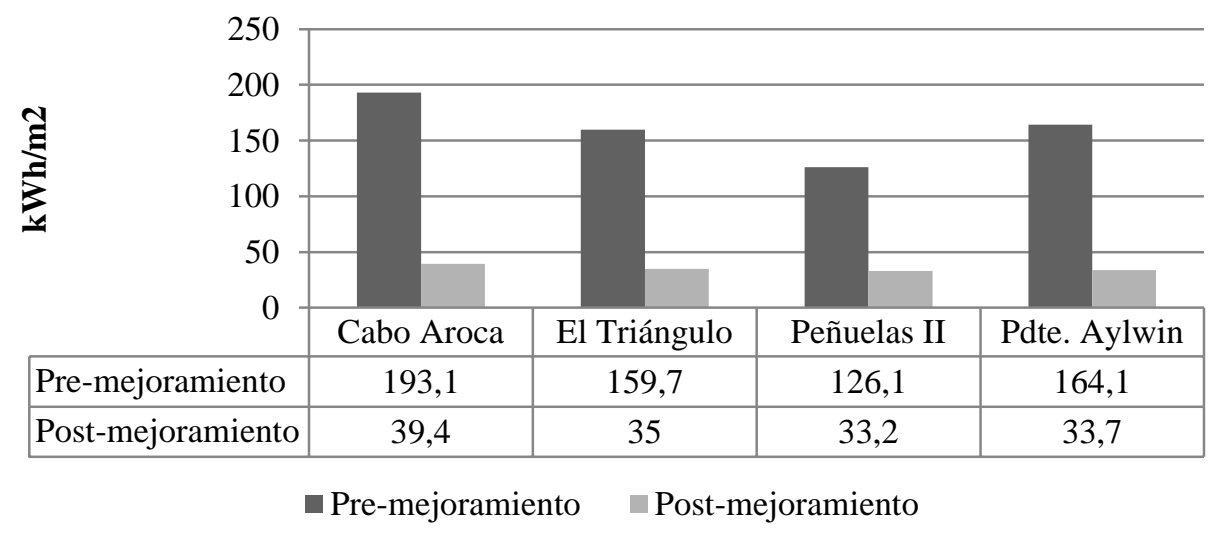

54 Martínez, P. W.; Kelly, M. T. 
Figura 5 - (Superior) Gráfico de demandas energéticas a partir del caso real con estrategias de mejoramiento propuestas - se destacan caso real, base E1 y E2 y sus respectivos mejores casos; (Inferior) Tabla con valores de factores utilizados en cada estrategia

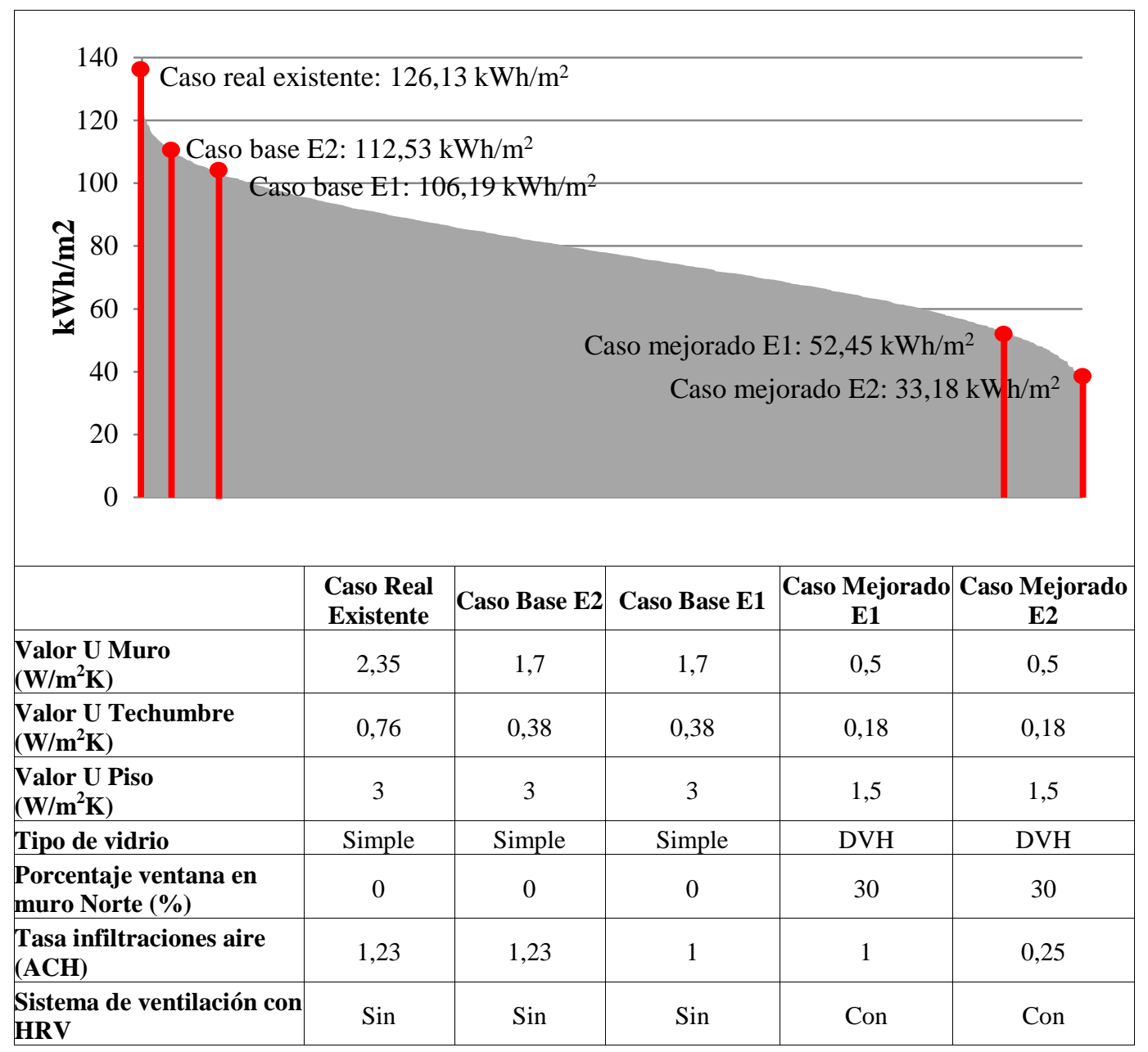

Figura 6 - Comparación de calificación energética según demanda energética para calefacción de la vivienda Peñuelas II con 1 ACH (izquierda) y distinto niveles (derecha) de tasa de infiltración

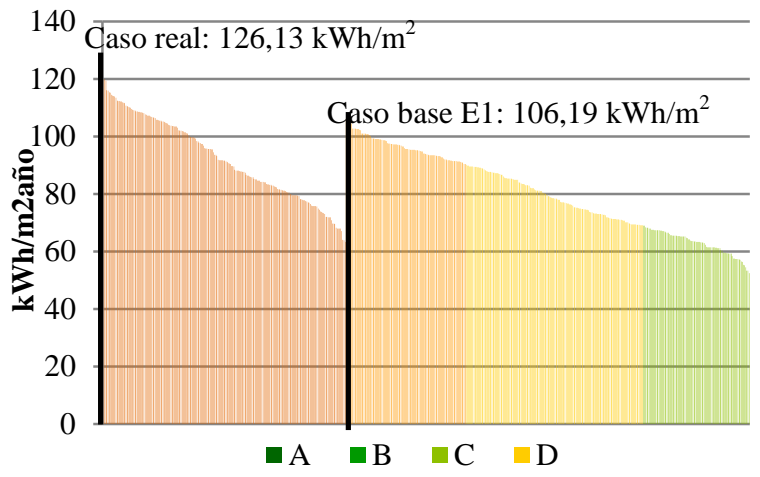

Ninguno de los casos arroja respuestas calificadas como G (más deficiente). La diferencia es que solo las estrategias con variadas propuestas de hermeticidad al aire de la envolvente pueden ser calificadas como A o B, dependiendo de la

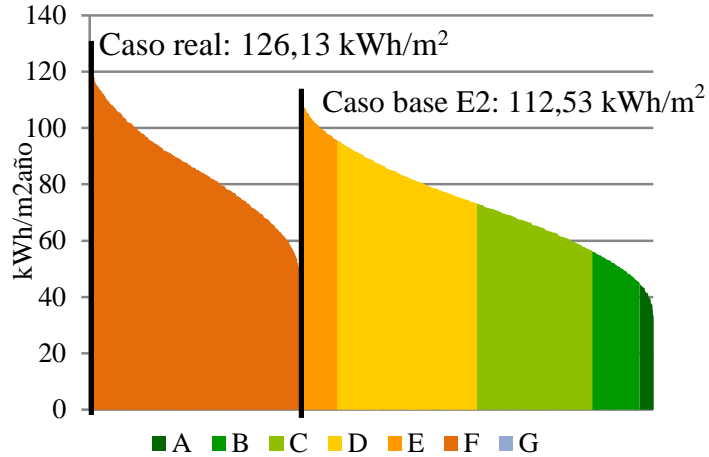

incorporación de otras mejoras. En cambio con 1 $\mathrm{ACH}$ como tasa de infiltración constante, y sin mejoras en ésta, no es posible ser altamente eficiente, ya que sólo alcanza calificación C (Figura 6). La menor demanda obtenida a partir de 
las distintas propuestas de mejoramiento a la vivienda inicial es de $33,18 \mathrm{kWh} / \mathrm{m}^{2}$ año, es decir disminuye $74 \%$ la demanda energética.

Comparando los niveles de parámetros más frecuentados dentro de cada rango de calificación (Tabla 2), se obtiene que los niveles deben mantenerse exactamente igual para no calificar como energéticamente eficientes $(\mathrm{F})$, y por el contrario, deben cumplir con los valores más exigentes de los propuestos, para obtener la mejor categorización (A). La tasa de infiltración de aire y todos los otros valores, exceptuando valor $\mathrm{U}$ de muro y techo, deben ser iguales a los valores actuales para alcanzar el estándar E. Es decir el esfuerzo de mejoramiento solo se debe realizar en dos parámetros de la envolvente, pero cabe destacar que la disminución de demanda energética no es muy significativa ( $10 \%$ menos), aunque si cumpliría con la RT. Se percibe que la utilización del sistema de ventilación con recuperación de calor se revela cuando la tasa de infiltración de aire es igual, o inferior, a $0,75 \mathrm{ACH}$.

Para calificar dentro del rango A al D (mayormente eficientes), se concluye que los niveles debiesen ser (según Tabla 2): (a) valor $\mathrm{U}$ de muro disminuye a $0,5 \mathrm{~W} / \mathrm{m}^{2} \mathrm{~K}$, o bien 1,7 si se quiere calificar con $\mathrm{D}$;

(b) valor $\mathrm{U}$ de techumbre debe ser $0,18 \mathrm{~W} / \mathrm{m}^{2} \mathrm{~K}$, o 0,38 para calificar D;

(c) valor $\mathrm{U}$ de piso debe disminuir a la mitad: 1,5 $\mathrm{W} / \mathrm{m}^{2} \mathrm{~K}$;

(d) las ventanas con doble vidrio hermético y con superficie vidriada extra en el muro norte de 20 a $30 \%$;

(e) la tasa de infiltraciones de aire debe ser disminuida a un rango entre 0,25 y $1 \mathrm{ACH}$; y

(f) se hace imprescindible el uso de un sistema de ventilación con recuperación de calor para estar dentro de A-C.

Estas estrategias se conocen desde mayorías absolutas por rango de calificación, es decir no se conoce con exactitud el nivel de integración entre ellos para el resultado final. Para poder conocer las tendencias de influencias de los parámetros propuestos de forma holística, se realiza un nuevo análisis estadístico y multifactorial, basado en el método Box-Cox.

Tabla 2 - Combinaciones de factores más frecuentados en cada rango de la Calificación energética para Peñuelas II con $1 \mathrm{ACH}$ y $\mathrm{ACH}$ real

\begin{tabular}{|c|c|c|c|c|c|c|c|c|}
\hline & & $\mathbf{A}$ & B & $\mathrm{C}$ & D & $\mathbf{E}$ & $\mathbf{F}$ & G \\
\hline \multirow{2}{*}{$\begin{array}{l}\text { Valor U Muro } \\
\left(\mathbf{W} / \mathbf{m}^{2} \mathbf{K}\right)\end{array}$} & E1 & - & - & 0,5 & 0,5 & 1,7 & 2,35 & - \\
\hline & E2 & 0,5 & 0,5 & 0,5 & 1,7 & 1,7 & 2,35 & - \\
\hline \multirow{2}{*}{$\begin{array}{l}\text { Valor U } \\
\text { Techumbre } \\
\left(\mathbf{W} / \mathbf{m}^{2} \mathbf{K}\right)\end{array}$} & E1 & - & - & 0,18 & 0,18 & 0,38 & 0,76 & - \\
\hline & E2 & 0,18 & 0,18 & 0,18 & 0,38 & 0,38 & 0,76 & - \\
\hline \multirow{2}{*}{$\begin{array}{l}\text { Valor U Piso } \\
\left(\mathbf{W} / \mathbf{m}^{2} \mathbf{K}\right)\end{array}$} & E1 & - & - & 1,5 & 1,5 & 3 & 3 & - \\
\hline & E2 & 1,5 & 1,5 & 1,5 & 3 & 3 & $1,5 / 3$ & - \\
\hline \multirow{2}{*}{ Tipo de vidrio } & E1 & - & - & DVH & Simple & Simple & Simple & - \\
\hline & E2 & DVH & $\mathrm{DVH}$ & DVH & Simple & Simple & $\mathrm{S}$ & - \\
\hline \multirow{2}{*}{$\begin{array}{l}\text { Porncentaje de } \\
\text { ventana en muro } \\
\text { Norte }(\%)\end{array}$} & E1 & - & - & 30 & $0 / 20$ & 20 & 20 & - \\
\hline & E2 & 30 & $0 / 20$ & 30 & 20 & 0 & $0 / 20 / 30$ & - \\
\hline \multirow{2}{*}{$\begin{array}{l}\text { Tasa de } \\
\text { infiltraciones } \\
(\mathrm{ACH})\end{array}$} & E1 & - & - & 1 & 1 & 1 & 1 & - \\
\hline & E2 & 0,25 & 0,25 & 0,75 & 1 & 1,23 & $\begin{array}{l}\text { Todas por } \\
\text { igual }\end{array}$ & - \\
\hline \multirow{2}{*}{$\begin{array}{l}\text { Uso de sistema de } \\
\text { ventilación con } \\
\text { HRV }\end{array}$} & E1 & - & - & Con & Con & Sin & Sin & - \\
\hline & E2 & Con & Con & Con & Sin & Sin & Sin & - \\
\hline
\end{tabular}




\section{Comprobación estadística de parámetros relevantes}

Los valores de un parámetro están distribuidos de tal forma que muestran una clara asimetría, dificultando el estudio, ya que los métodos estadísticos tradicionales suelen emplear promedios y desviaciones estándar (SCHUSCHNY; SOTO, 2009). Para poder corregir esta asimetría, se utiliza el método BoxCox (Tabla 3), que entrega "visibilidad" de aquellos factores que son más relevantes, y otros desechables (HEISELBERG et al., 2010), aumentando la exactitud del análisis de sensibilidad. Este análisis multifactorial mejora la eficiencia del proceso de diseño, además de ser muy útil en la optimización del desempeño del edificio. A diferencia de otros métodos, este permite relacionar todos los criterios de desempeño, para conocer la interacción de aquellos que influyen en el objetivo buscado.

En la Figura 7 se muestra el diagrama que representa la distribución acumulada de las observaciones estandarizadas. El propósito de este tipo de gráficos es evaluar la bondad de ajuste de la distribución de los niveles especificados a una distribución determinada. Permite conocer cómo se distribuyen los niveles a partir de las variables de respuestas, que corresponden a 7 niveles. El 1 equivale la letra A y el 7 a la calificación G, cada uno de estos valores se obtuvo a partir de un promedio de los rangos adquiridos desde el análisis con la $\mathrm{CE}$.

Los demás niveles de parámetros se ordenan en base a la influencia que tienen en las 7 respuestas, por lo tanto los datos no se ajustan a la distribución normal arrojada por las respuestas de demanda de energía. Debido a esto, y a que las respuestas no tienen un carácter lineal, no se observa que los niveles de los factores estén cercanos a la respuesta 1 , sino que la mayoría tienden a la respuesta con que están relacionadas, por ejemplo puede existir un nivel que este cercano al 7, lo que significa que aquel nivel está asociado a una alta demanda energética. En cambio, aquellos parámetros cercanos al centro (0), tienen muy baja relevancia en las respuestas, siendo consideradas como poco influyentes. La nomenclatura utilizada, para poder interpretar la Figura 7, se resume en la Tabla 4.

Para el caso de estudio Peñuelas II, se obtuvieron los siguientes parámetros, como los más óptimos para la disminución de demanda de calefacción (Figura 7):

(a) tasa de infiltración de aire entre 0,25 y 0,75 $\mathrm{ACH}$;

(b) valor $\mathrm{U}$ del muro más bajo que $0,8 \mathrm{~W} / \mathrm{m}^{2} \mathrm{~K}$; y

(c) uso de un sistema de ventilación con recuperación de calor.

Se observa que los valores reales de los niveles están asociados a las más altas demandas de energía, pero sobre todo una alta tasa de infiltración de aire, el valor U real del muro y el no considerar un sistema de ventilación con recuperación de calor, lo cual es lógico ya que son las medidas opuestas a las necesarias para el mejoramiento energético-ambiental de la vivienda.

Tabla 3 - Nomenclatura para estudio de Box-Cox

\begin{tabular}{|c|c|c|}
\hline Parámetro & Nivel & Clasificación \\
\hline \multirow[b]{2}{*}{ Valor U muro } & $0,5 \mathrm{~W} / \mathrm{m}^{2} \mathrm{~K}$ & D1 \\
\hline & \multicolumn{2}{|c|}{$\begin{array}{l}\text { A medida que empeora la transmitancia térmica, } \\
\text { aumenta el número de D (D2, D3, etc.) }\end{array}$} \\
\hline \multirow[b]{2}{*}{ Valor U Techumbre } & $0,18 \mathrm{~W} / \mathrm{m}^{2} \mathrm{~K}$ & $\mathrm{~F} 1$ \\
\hline & \multicolumn{2}{|c|}{$\begin{array}{l}\text { A medida que empeora la transmitancia térmica, } \\
\text { aumenta el número de } \mathrm{F}(\mathrm{F} 2, \mathrm{~F} 3 \text {, etc.) }\end{array}$} \\
\hline \multirow[t]{2}{*}{ Valor U Piso } & $1,5 \mathrm{~W} / \mathrm{m}^{2} \mathrm{~K}$ & E1 \\
\hline & $3 \mathrm{~W} / \mathrm{m}^{2} \mathrm{~K}$ & E2 \\
\hline \multirow{2}{*}{$\begin{array}{l}\text { Tipo de Vidrio de } \\
\text { Ventana }\end{array}$} & Simple & G1 \\
\hline & Doble Vidrio Hermético & $\mathrm{G} 2$ \\
\hline \multirow{2}{*}{$\begin{array}{l}\text { Porcentaje de Superficie } \\
\text { Vidriada }\end{array}$} & $0 \%$ & $\mathrm{~A} 1$ \\
\hline & \multicolumn{2}{|c|}{$\begin{array}{l}\text { A medida que aumenta el porcentaje, aumenta el } \\
\text { número de } \mathrm{A}(\mathrm{A} 2, \mathrm{~A} 3 \text {, etc.) }\end{array}$} \\
\hline \multirow{2}{*}{$\begin{array}{l}\text { Tasa de Infiltración de } \\
\text { Aire }\end{array}$} & $0,25 \mathrm{ACH}$ & $\mathrm{C} 1$ \\
\hline & \multicolumn{2}{|c|}{$\begin{array}{l}\text { A medida que aumenta la tasa de infiltración de aire, } \\
\text { aumenta el número de } \mathrm{C}(\mathrm{C} 2, \mathrm{C} 3 \text {, etc. })\end{array}$} \\
\hline \multirow{2}{*}{$\begin{array}{l}\text { Sistema de Ventilación } \\
\text { HRV }\end{array}$} & Sin HRV & B1 \\
\hline & Con HRV & $\mathrm{B} 2$ \\
\hline
\end{tabular}


Figura 7 - Diagrama de normalización principal por variable de Peñuelas II

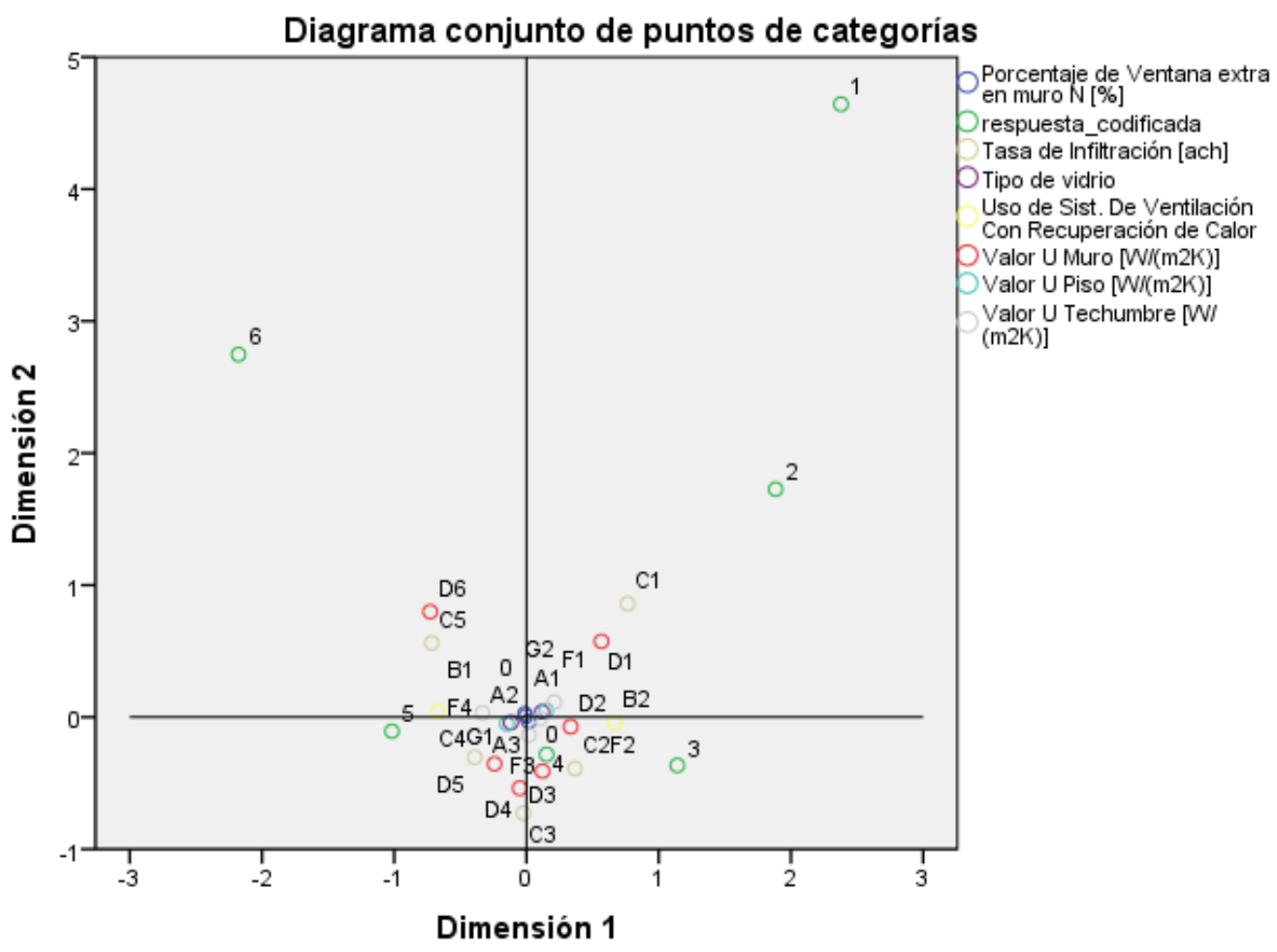

El tipo de vidrio y el porcentaje de superficie transparente en el muro norte, se acercan a 0 , siendo consideradas como irrelevantes, pero es necesario tener reparo en que las ventanas si son muy importantes cuando se busca reducir el riesgo de condensación superficial, ya que según el análisis de este desempeño intermedio, es necesario tener menor transmitancia térmica en el vidrio para disminuir este riesgo, y eso no se puede lograr con un vidrio simple como el que tienen las viviendas actualmente.

En todos los casos se observa que el uso del sistema de ventilación con recuperación de calor siempre va asociado al desempeño de hermeticidad al aire de la envolvente, donde la tasa de infiltración de aire debe ser inferior a 0,75 ACH.

Más allá de considerar niveles propuestos, tales como 0,25 ACH de tasa de infiltración de aire, $>0,8 \mathrm{~W} / \mathrm{m}^{2} \mathrm{~K}$ de transmitancia térmica en el muro y el uso de un sistema de ventilación con HRV, como los necesarios para disminuir la demanda, es preciso realizar el mejoramiento energéticoambiental con el uso de los parámetros influyentes de una manera integral; es decir no basta con realizar el mejoramiento con el primer factor de influencia solamente.

\section{Análisis en función del confort térmico}

El objetivo de esta evaluación es conocer cuánto mejora el confort térmico al interior de la vivienda, mostrando la cantidad de horas en que la temperatura interior de la vivienda está dentro y fuera del rango de confort.

Basado en los parámetros determinados como más relevantes en el análisis en función de la demanda energética, se evalúa el mejoramiento de la vivienda considerando solo estas tres variables de manera integral, manteniendo el resto de parámetros en su estado actual. El sistema de ventilación con HRV tiene un alto costo en Chile, debido a su baja demanda en el país. Entonces, se proponen dos packs de mejoramientos (P1 y P2), donde uno considera el uso del sistema y el otro no, manteniendo fijas las otras dos estrategias.

Al considerar el uso de un sistema de ventilación con HRV, la demanda energética para calefaccionar la vivienda disminuye $57,3 \%$ de lo inicial (Tabla 4). En cambio, la vivienda con el pack que no usa un sistema de ventilación con HRV disminuye su demanda energética en 38,7\%. Esto significa que el uso de tal sistema tiene un efecto de, al menos, $20 \%$ más eficiente en la variable de demanda de energía. Por ende, el sistema no solo asegura eficiencia energética, sino que asegura buena calidad de aire interior y con 
caudales de renovación de aire mínimos, teniendo siempre en consideración que la hermeticidad al aire de la envolvente debe ser alta. Además, en la etapa de diagnóstico los usuarios de esta tipología de vivienda declararon percibir humedad superficial en el interior, lo cual decrecería con este sistema y con la disminución de transmitancia térmica en el muro.

Tal como se observó a partir del análisis de relevancia de parámetros, el cambio de vidrio en ventanas no tiene mayor aporte en la disminución de energía (Tabla 5), aumentando la eficiencia solo $1,5 \%$.

Con el mejoramiento $\mathrm{P} 1$, se obtiene que durante la temporada cálida, el porcentaje dentro de la zona de confort térmico aumenta poco, ya que siguen existiendo horas por debajo de la zona de confort térmico, pero el riesgo de sobrecalentamiento desaparece (Tabla 6). En la Figura 8 se observa que la temperatura mínima alcanzada en la temporada cálida aumenta de 12 a $16^{\circ} \mathrm{C}$. La mayor parte del tiempo en que las temperaturas están por debajo de la zona de confort corresponde a los meses de octubre y noviembre, donde las temperaturas exteriores no son muy altas.

El confort térmico mejora considerablemente en la temporada fría. En un principio la vivienda mostró solo $8 \%$ de las horas en confort $y$ ahora corresponden a 77,8\% (Tabla 7). En la Figura 9, se observa que la temperatura inferior es $16^{\circ} \mathrm{C}$, siendo ampliamente diferente a la inicial, donde la temperatura más fría correspondía a $8^{\circ} \mathrm{C}$. Además la temperatura ambiente de la vivienda mejorada es más constante, no sufre grandes oscilaciones, revelando solo diferencias en abril y septiembre, donde se registran mayores temperaturas en el exterior.

Tabla 4 - Demanda energética por calefacción en vivienda Peñuelas II inicial y mejorada con los desempeños intermedios propuestos

\begin{tabular}{cc}
\hline & $\begin{array}{c}\text { Demanda Energética } \\
\left(\mathbf{k W h} / \mathbf{m}^{\mathbf{2}}\right)\end{array}$ \\
\hline Peñuelas II Inicial & $126,13 \mathrm{kWh} / \mathrm{m}^{2}$ \\
Peñuelas II Con Sistema de Ventilación HRV (P1) & $53,9 \mathrm{kWh} / \mathrm{m}^{2}$ \\
Peñuelas II Sin Sistema de Ventilación HRV (P2) & $77,31 \mathrm{kWh} / \mathrm{m}^{2}$ \\
\hline
\end{tabular}

Tabla 5 - Demanda energética por calefacción en vivienda Peñuelas II inicial y mejorada con los desempeños propuestos más el cambio de vidrio simple a doble vidrio hermético (DVH)

\begin{tabular}{cc}
\hline & Demanda Energética $\left(\mathbf{k W h} / \mathbf{m}^{2}\right)$ \\
\hline Peñuelas II Inicial & $126,13 \mathrm{kWh} / \mathrm{m}^{2}$ \\
P1 + DVH & $49,8 \mathrm{kWh} / \mathrm{m}^{2}$ \\
P2 + DVH & $73,22 \mathrm{kWh} / \mathrm{m}^{2}$ \\
\hline
\end{tabular}

Tabla 6 - Porcentaje de horas bajo, dentro y sobre la zona de confort en temporada Cálida para la vivienda Peñuelas II inicial y mejorada

\begin{tabular}{lccc}
\hline & $\begin{array}{c}\text { Bajo Rango Confort (8- } \\
\left.\mathbf{2 0}^{\circ} \mathbf{C}\right)\end{array}$ & $\begin{array}{c}\text { Rango de Confort } \\
\left(\mathbf{2 0 - 2 5} \mathbf{C}^{\circ} \mathbf{C}\right)\end{array}$ & $\begin{array}{c}\text { Sobre Rango Confort } \\
\left(\mathbf{2 5 - 3 0} \mathbf{C}^{\circ} \mathbf{C}\right)\end{array}$ \\
\hline Peñuelas II Inicial & $62,0 \%$ & $37,5 \%$ & $0,5 \%$ \\
Peñuelas II Mejorada (P1) & $52,0 \%$ & $48,0 \%$ & $0 \%$ \\
\hline
\end{tabular}


Figura 8 - Comparación de gráficos de temperatura dentro y fuera de la zona de confort en temporada cálida (Izquierda: Peñuelas Inicial; y Derecha: Peñuelas mejorada P1)
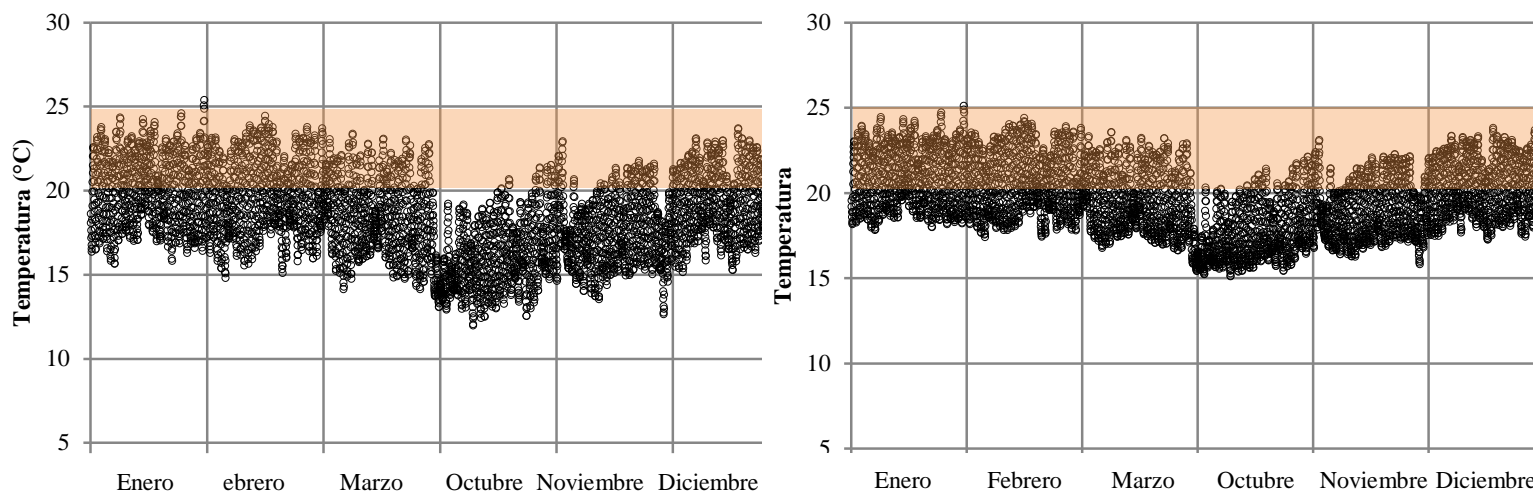

Tabla 7 - Porcentaje de horas bajo, dentro y sobre la zona de confort en Temporada Fría para la vivienda Peñuelas II inicial y mejorada

\begin{tabular}{cccc}
\hline & $\begin{array}{c}\text { Bajo Rango Confort } \\
\left(\mathbf{4 - 1 8}^{\circ} \mathbf{C}\right)\end{array}$ & $\begin{array}{c}\text { Rango de Confort } \\
\left(\mathbf{1 8 - 2 3}^{\circ} \mathbf{C )}\right.\end{array}$ & $\begin{array}{c}\text { Sobre Rango Confort } \\
\left(\mathbf{2 3 - 2 8} \mathbf{C}^{\circ}\right.\end{array}$ \\
\hline Peñuelas II Inicial & $93,0 \%$ & $7,0 \%$ & $0 \%$ \\
\hline Peñuelas II mejorada (P1) & $22,2 \%$ & $77,8 \%$ & $0 \%$ \\
\hline
\end{tabular}

Figura 9 - Comparación de gráficos de temperatura dentro y fuera de la zona de confort durante la temporada fría (Izquierda: Peñuelas II Inicial; y Derecha: Peñuelas II mejorada P1)

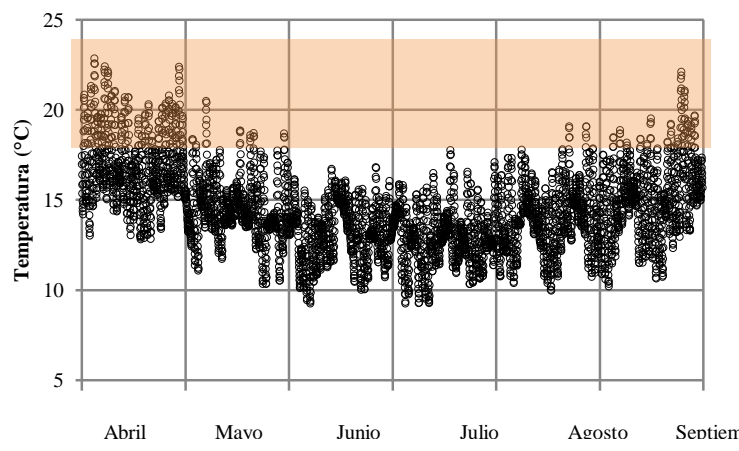

\section{Conclusiones}

Al proponer estrategias de mejoramiento en viviendas existentes, se sugiere medir ciertas propiedades físicas de la construcción como diagnóstico, para calibrar el modelo en las simulaciones, y así obtener resultados más reales.

Desde las simulaciones se pueden conocer los desempeños a considerar, cuyos objetivos deben establecer requisitos mínimos para poder desarrollar estrategias de mejoramientos, considerando además sus indicadores y mediciones necesarias. Los desempeños evaluados y propuestos como criterios a mejorar, son fenómenos multidimensionales, motivo por el cual

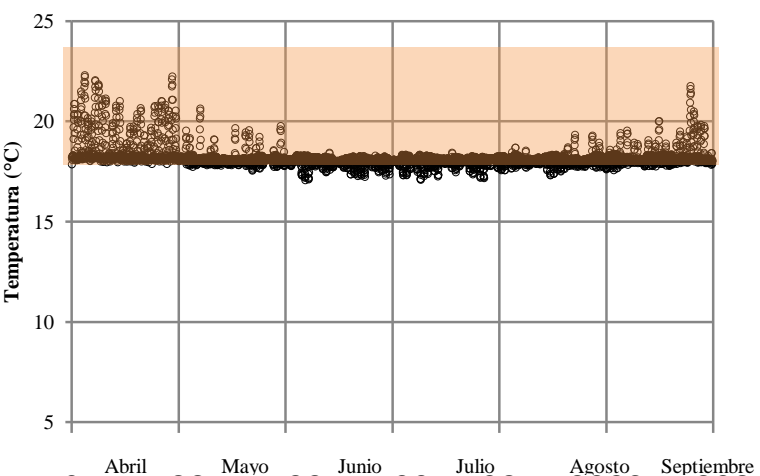

el diagnóstico y las estrategias deben ser integrales, entendiendo que las propuestas de mejoramiento energético-ambiental se deben realizar de forma complementaria entre factores, y no individual.

Del análisis de demanda energética por calefacción, se obtuvo que el estudio de transformación paramétrico es más sensible al carácter integral que propone esta investigación, determinando que independiente de ser un parámetro muy influyente en la disminución de demanda energética, no basta con mejorarlo de manera individual, sino que es el mejoramiento de todos los parámetros influyentes lo que entrega mejores resultados. 
Establecer nuevos y más exigentes estándares permite superar problemas de eficiencia energética y confort térmico al interior de las viviendas. En el caso de estudio se observa que utilizando las estrategias de mejoramiento más exigentes, se pueden alcanzar demandas energéticas inferiores a $40 \mathrm{kWh} / \mathrm{m}^{2}$, es decir $80 \%$ más eficiente que la demanda actual. Además se detectó la importancia del carácter integral en un mejoramiento, al existir ciertas combinaciones de criterios de desempeños que no cumplían con la Reglamentación Térmica, siendo calificadas como $\mathrm{G}$ o F, pero que lograban disminuir $65 \%$ la demanda energética para calefaccionar, siendo más eficientes que otros casos que si cumplen con RT (califican como E, D o C) pero que demandan más energía. Esto permite concluir que no basta con concentrar esfuerzos en el cumplimiento de una normativa, sino que de preferencia hay que poner atención en soluciones integrales de mejoramiento para alcanzar mayores beneficios.

De los desempeños identificados, como más relevantes en la disminución de energía, se observa que el valor de transmitancia térmica del muro debiera ser al menos la mitad del exigido actualmente por la normativa chilena para la zona estudiada, variando entre 0,5 a $0,8 \mathrm{~W} / \mathrm{m}^{2} \mathrm{~K}$, asegurando además un bajo riesgo de condensación superficial. La infiltración de aire, que no es considerada en la reglamentación nacional para viviendas, tiene alta influencia en la disminución de la demanda energética y en el mejoramiento del confort térmico de los casos de estudio. De la comparación hecha en base al caso real, considerando una tasa de infiltración de 1 $\mathrm{ACH}$ y el mismo caso con las propuestas de mejoramiento en la hermeticidad al aire, se determina que utilizando el valor por defecto propuesto por la Calificación Energética, es imposible alcanzar la calificación A. Debido a que por mayor que sea el esfuerzo por mejorar otros desempeños, como la aislación térmica por ejemplo, se asume perdida de calor por infiltraciones de aire. Cabe destacar que este tipo de mediciones son onerosas y requieren de personas calificadas, por lo que se justifica seleccionar tipologías representativas en el parque construido para poder ampliar los datos obtenidos, y así considerar este desempeño de alta pertinencia al momento de busca estos tipos de mejoramiento en a vivienda.

Al realizar, de manera teórica, el mejoramiento energético-ambiental en las viviendas existentes, sin ninguna estrategia en particular, sino que utilizando los estándares más influyentes obtenidos desde el estudio, se alcanzan beneficios en todos los objetivos finales de desempeños propuestos.
Primero, la eficiencia energética por calefacción es lograda utilizando las tres estrategias más influyentes (valor u muro, infiltración de aire y sistema de ventilación con HRV) y manteniendo el resto de parámetros igual que en su estado actual, disminuyendo la demanda energética en casi $60 \%$. $\mathrm{Si}$ bien este beneficio es el que menos incidencia tiene en el presupuesto familiar de una vivienda social, debido a su alta vulnerabilidad y a sus bajos gastos destinados a calefacción, si se obtienen beneficios por términos de temperatura. Para la temporada fría, los usuarios manifestaron el requerimiento de aumentar la temperatura al interior, situación que post-mejoramiento se logra, incrementando 10 veces la cantidad de horas dentro de la zona de confort térmico, logrando prescindir del uso de un sistema de calefacción por largas horas, favoreciendo la situación de las familias que se encuentran bajo la línea de pobreza. Considerando la percepción de usuario, se asume que las estrategias en verano serían bien recibidas, ya que la temperatura operativa existente es considerada como neutral y esperaban que ante cualquier cambio se mantuviera igual.

Con respecto al tercer objetivo de desempeño, calidad del aire interior, se asume un mejoramiento desde la etapa de diagnóstico, al establecer una tasa de aire mínima por metro cuadrado. Este objetivo, al igual que los otros, visto bajo una filosofía prestacional debe cumplir con lo exigido incorporando cualquier estrategia pero que asegure el caudal mínimo. Hay que tener en consideración que al ser disminuida la infiltración de aire no deseado, el caudal de renovación de aire disminuye, haciendo necesario incorporar una estrategias que asegure el caudal de manera forzada.

El valor del parámetro que se usará dependerá de cada caso de estudio a mejorar, pero se concluye que los parámetros influyentes se repiten en los cuatro casos de estudio, por lo que, independiente del valor asignado (dentro de un rango aceptable propuesto) se debiese incorporar el mejoramiento integral de dichos desempeños intermedios a viviendas sociales de este tipo y construidas en clima templado-cálido con estación seca corta.

\section{Referencias}

\section{AMERICAN SOCIETY OF HEATING, REFRIGERATING AND AIR CONDITIONING ENGINEERS. ANSI/ASHRAE 55: thermal} environmental conditions for human occupancy. Atlanta, 2010. 
CAUBERG-HUYGEN CONSULTING ENGINEERS. ECOLISH: energy exploitation and performance contracting for low income and social housing. Netherlands: IEE, 2009.

COMISIÓN NACIONAL DE ENERGÍA. Balance Nacional de Energía. Santiago: Gobierno de Chile, 2011.

FIGUEROA, R. et al. Air Infiltration in Chilean Housing: a baseline determination. In: PLEA2013, 29., Munich, 2013. Proceedings... Munich, 2013.

HEISELBERG, P. et al. Application of Sensitivity Analysis in Design of Sustainable Buildings. Renewable Energy, v. 34, n. 9, p. 2030-2036, 2010.

HEO, Y.; CHOUDHARY, R.; AUGENBROE, G. Calibration of Building Energy Models For Retrofit Analysis Under Uncertainty. Energy and Buildings, v. 47, n. 4, p. 550-560, 2012.

HONG, S. et al. A Field Study of Thermal Comfort in Low-Income Dwellings in England Before and After Energy Efficient Refurbishment. Building and Environment, v. 44, p. 1228-1236, 2009.

HOPFE, C.; HENSEN, J. Uncertainty Analysis in Building Performance Simulation For Design Support. Energy and Buildings, v. 43, n. 10, p. 2798-2805, 2011.

HOWDEN-CHAPMANA, P. et al. Retrofitting Houses With Insulation to Reduce Health Inequalities: aims and methods of a clustered, randomised community-based trial. Social Science \& Medicine, v. 61, n. 12, p. 2600-2610, 2005.

INSTITUTO NACIONAL DE ESTADÍSTICA. Anuario de Edificación Comunal: región del BíoBío. 13. ed. 2010. Concepción: Gobierno de Chile, 2010.

INTERNATIONAL ENERGY AGENCY. $\mathrm{CO}_{2}$ Emissions from Fuel Combustion - Highlights. Paris: OECD/IEA, 2010. 128p.

INZUNZA, J. Clasificación de los Climas de Köppen. Ciencia Ahora, v.15, n. 8, p. 131-156, 2005.

LAM, J. et al. Principal Component Analysis and Long-Term Building Energy Simulation Correlation. Energy Conversion and Management, v. 51, n. 2, p. 135-139, 2010.
LEE, A.; BARRETT, P. Performance Based

Building: first international state-of-the-art report. University of Salford, UK For CIB Development Foundation, PeBBu Thematic Network, 2003.

MERCADO, M.; ESTEVES, A.; FILIPPÍN, C. Comportamiento Térmico-Energético de Una Vivienda Social de la Ciudad de Mendoza, Argentina. Ambiente Construido, Porto Alegre, v. 10, n. 2, p. 87-100, abr./jun. 2010.

MINISTERIO DE VIVIENDA Y URBANISMO. Artículo 4.1.10. Manual de Aplicación. Reglamentación Térmica. Ordenanza general de urbanismo y construcciones. Santiago, 2006.

MONTGOMERY, D. Diseño y Análisis de Experimentos. 2. ed. Mexico: Limusa, 2003.

MWASHA, A.; WILLIAMS, G.; IWARO, J. Modeling the Performance of Residential Building Envelope: the role of sustainable energy performance indicators. Energy and Buildings, v. 43, n. 9, p. 2108-2117, 2011.

NORDIC COMMITTEE ON BUILDING REGULATIONS. Programme of Work for the NKB Structure for Building Regulations. Stockholm, 1978. Report No 34.

OLOFSSON, T.; ANDERSSON, S.; SJÖGREN, J. Building Energy Parameter Investigations Based on Multivariate Analysis. Energy and Buildings, v. 41, n. 1, p. 71-80, 2009.

SCHUSCHNY, A.; SOTO, H. Guía Metodológica: diseño de indicadores compuestos de desarrollo sostenible. Santiago: Publicaciones de Naciones Unidas, 2009.

TAVARES, P.; MARTINS, A. Energy efficient building design using sensitivity analysis-A case study. Energy and Buildings, v. 39, n. 1, p. 2331, 2007.

WEGERTSEDER, P. Diagnosis of Physical Quality of Existing Social Housing without Thermal Considerations in Chile. In: PLEA2013, 29., Munich, 2013. Proceedings... Munich, 2013.

WILSON, C.; SMITH, B.; DUNN, P. EcoHomes $\mathrm{XB}$ : a guide to the EcoHomes methodologhy for existing buildings. Sustainable Homes LTD, 2007.

\section{Agradecimientos}

Esta investigación es parte de la tesis doctoral de la autora, que se desarrolló en la Universidad del Bío-Bío en Concepción, Chile. 


\section{Paulina Wegertseder Martínez}

Departamento de Diseño y Teoría de la Arquitectura | Universidad del Bío-Bío | Av. Collao 1202 | Concepcion - Chile | 4051381 |

Tel.: +56 (41) 311-1440 | E-mail: pwegertseder@ubiobio.cl

\section{Maureen Trebilcock Kelly}

Departamento de Diseño y Teoría de la Arquitectura | Universidad del Bío-Bío | Tel.: +56 (41) 311-1442 | E-mail: mtrebilc@ubiobio.cl

\section{Revista Ambiente Construído}

Associação Nacional de Tecnologia do Ambiente Construído

Av. Osvaldo Aranha, $99-3^{\circ}$ andar, Centro

Porto Alegre - RS - Brasil

CEP 90035-190

Telefone: +55 (51) 3308-4084

Fax: +55 (51) 3308-4054

www.seer.ufrgs.br/ambienteconstruido

E-mail: ambienteconstruido@ufrgs.br 